\title{
Development of light-weight spherical mirrors for RICH detectors
}

\author{
F.C.D. Metlica
}

H.H. Wills Physics Laboratory, Department of Physics, University of Bristol, Tyndall Avenue, Bristol, BS8 1TL, UK

\section{On behalf of the LHCb Collaboration}

\section{A R T I C L E I N F O}

Keywords:

$\mathrm{RICH}$

$\mathrm{LHCb}$

Light-weight mirrors

Carbon-fiber

Beryllium

\begin{abstract}
A B S T R A C T
Glass-coated beryllium and carbon-fiber light-weight spherical mirrors, with radii of curvature of $\sim 2700 \mathrm{~mm}$, have been successfully developed and tested as part of the LHCb RICH experimental programme. The low mass mirror is necessary to minimize the amount of material within the LHCb spectrometer acceptance, with a requirement of less than $2 \%$ of a radiation length. Both technologies are suitable for light-weight mirror applications. The R\&D and characterization of the mirrors are reported. (c) 2008 Elsevier B.V. All rights reserved.
\end{abstract}

\section{Introduction}

Light-weight mirrors are required in RICH detectors whenever the material budget needs to be minimized to reduce particle interactions with the mirror material. Two promising light-weight technologies have been developed for the spherical mirrors of the RICH 1 detector of the LHCb experiment [1]: glass-coated beryllium and carbon-fiber. After a successful R\&D programme in both technologies, the carbon-fiber technology was chosen. Three important parameters define the mirror optical quality: reflectivity, radius of curvature $(R)$ and the average geometrical quality $D_{0}$. The parameter $D_{0}$ is defined as the diameter of the circle at the mirror center of curvature ( $\mathrm{CoC}$ ) which contains $95 \%$ of the reflected light intensity from a point source placed at the CoC. The two mirror technologies have been characterized at CERN and both must satisfy the following requirements:

- light-weight: radiation length $<2 \% X_{0}$ and a nuclear interaction length $<1 \% \lambda_{\mathrm{I}}$;

- radiation tolerant: no significant degradation of the mirror properties up to $10 \mathrm{kGy}$ absorbed dose, equivalent to 10 years in the radiation environment of RICH 1 ;

- fluorocarbon compatibility: with the $\mathrm{C}_{4} \mathrm{~F}_{10} \mathrm{RICH} 1$ gas radiator;

- structural rigidity: the mirror does not deform under its own weight when supported;

- optical quality: $D_{0}<2.5 \mathrm{~mm}$, the radius of curvature is within $1 \%$ of its design value of $R=2700 \mathrm{~mm}$, and the average reflectivity is $\sim 90 \%$ in the $200-600 \mathrm{~nm}$ wavelength range.

\section{Glass-coated beryllium technology}

Beryllium $(Z=4)$ possesses unique properties ideal for lightweight applications, i.e. long radiation length, radiation hard, fluorocarbon compatibility, non-magnetic, light-weight and good rigidity. The principal disadvantages in using beryllium are the high manufacturing costs and its high toxicity which requires special safety measures during manufacturing and handling. Typical polished beryllium surfaces have an average surface roughness of $\sim 20-30 \mathrm{~nm}$ rms resulting in only $\sim 50 \%$ reflectivity in the visible and UV range. High reflectivity, $\sim 90 \%$ in the visible and UV, can be obtained by glass coating a beryllium substrate and then polishing the glass surface with standard optical methods and then applying an aluminium reflective film.

Three small sized glass-coated beryllium prototypes [2] have been manufactured in Russia ${ }^{1}$ and tested successfully at CERN. The first prototype is a circular flat mirror with a diameter of $95 \mathrm{~mm}$ and consists of a $10 \mathrm{~mm}$ thick beryllium substrate coated with a $1 \mathrm{~mm}$ glass layer, resulting in $3.6 \% X_{0}$ and $D_{0}<0.1 \mathrm{~mm}$. The second prototype is also circular, with a diameter of $280 \mathrm{~mm}$, consisting of a $5 \mathrm{~mm}$ thick beryllium substrate with a $1 \mathrm{~mm}$ glass layer coating and reinforced by a $20 \mathrm{~mm}$ thick rib-like structure, giving an average of $3.3 \% X_{0}$. The rib-like structure ensures high mirror rigidity. Its $D_{0}$ is $0.85 \mathrm{~mm}$ and radius of curvature $R=$ $7926 \mathrm{~mm}$ is close to the design value of $R=8000 \mathrm{~mm}$. The third prototype is rectangular shaped, $375 \mathrm{~mm} \times 300 \mathrm{~mm}$. It consists of a $6 \mathrm{~mm}$ thick beryllium substrate and a $0.3 \mathrm{~mm}$ glass coating, giving $1.9 \% X_{0}$. The measured $D_{0}$ is $0.41 \mathrm{~mm}$ and $R=1696 \mathrm{~mm}$ is very close to the design value of $1700 \mathrm{~mm}$.

As a result of the success of the small-sized prototypes, a fullsized beryllium prototype was designed and manufactured so

\footnotetext{
${ }^{1}$ Kompozit Joint Stock Company, Moscow.
} 

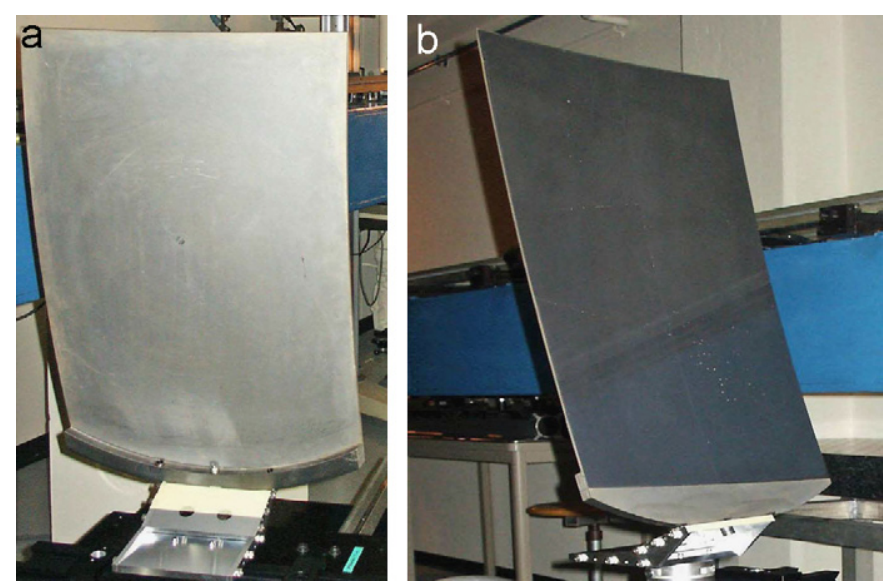

Fig. 1. The full-sized glass-coated beryllium prototype mirror supported at its rim as it would have been in RICH 1 , inclined at a $\sim 12^{\circ}$ angle with respect to the vertical. (a) Rear view, showing the beryllium rim at the bottom. (b) Front view, the dark area is the glass coating with no reflective film yet applied.

that, if also successful, it could have been used in RICH 1 [2]. The mirror dimensions are constrained by limitations in the manufacturing size of the beryllium blanks which are $800 \mathrm{~mm}$ in diameter. The full-sized prototype was designed to be as thin as possible but at the same time rigid enough not to deform under its own weight. The mirror consists of a $3 \mathrm{~mm}$ thick spherically shaped beryllium substrate $(R=2700 \mathrm{~mm})$ coated with a thin glass layer $(0.3-0.5 \mathrm{~mm})$, having a $20 \mathrm{~mm}$ thick beryllium rim at one edge to support it (see Fig. 1). The rim serves to bolt the mirror to the mirror support structure which lies outside the LHCb experimental acceptance. A FEA ${ }^{2}$ was performed to investigate the feasibility of the design, in particular the mirror support scheme with the induced stresses and distortions due to gravity, the natural vibration modes, and the effect on the $D_{0}$ caused by the gravity deflection of the mirror. A central point support on the beryllium rim (single bolt) was chosen as it gave the best FEA result i.e. a maximum deflection of the mirror due to gravity of $\sim 160 \mu \mathrm{m}$ and only a small effect, $<0.3 \mathrm{~mm}$, on the $D_{0}$.

The full-sized prototype mirror was manufactured by Kompozit in collaboration with Vavilov ${ }^{3}$ while the beryllium blank was procured from Ulba. ${ }^{4}$ The blank was produced by placing beryllium powder into a disc-shaped die where the powder was vibrated to obtain a homogeneous distribution, while heat and pressure were applied to compress and consolidate the powder into a solid blank. At the same time a vacuum was applied to outgas and prevent the formation of air bubbles. The blank, an $800 \mathrm{~mm}$ diameter disc $20 \mathrm{~mm}$ thick, was machined at Kompozit down to the final shape and thickness of the mirror and repeatedly annealed to relieve stresses. After machining, the beryllium substrate was $\sim 4 \mathrm{~mm}$ thick and spherically shaped with a radius of curvature very close to its final value. The design value of $3 \mathrm{~mm}$ thickness was not reached because of the high risk in breaking the blank during machining. Three mount holes were drilled into the beryllium rim and titanium inserts ${ }^{5}$ were glued ${ }^{6}$ into them. The central insert was used to bolt the mirror onto a support frame, while pins were inserted into the two side inserts as a safety mechanism to prevent accidental rotations. The glass coating was performed at Vavilov. The glass was chosen to match the coefficient of thermal expansion of beryllium. Several glass

\footnotetext{
${ }^{2}$ Finite element analysis software, ANSYS.

${ }^{3}$ Vavilov State Optical Institute, St. Petersburg, Russia.

${ }^{4}$ Ulba, Ust-Kamenogorsk, Kazakhstan.

${ }^{5}$ Safety forbids any direct contact with beryllium.

${ }^{6}$ Propriety information: low outgassing radiation hard glue.
}

sheets were placed on the beryllium substrate front face, covering it. The glass was melted by placing the substrate into an oven at $\sim 600^{\circ} \mathrm{C}$, forming a single glass layer fused onto the beryllium substrate. The glass layer was then polished using standard optical methods, where the fine tuning of the mirror radius of curvature was achieved, i.e. by appropriately optimizing the thickness of the glass layer across the mirror, typically $0.3-0.5 \mathrm{~mm}$. The last step would have been the application of an aluminium reflective coating, however, the coating was not applied since all the main optical and geometrical properties could be measured without it.

The full-sized prototype was characterized at CERN and was found to be of good optical quality: $D_{0}=3.3 \mathrm{~mm}$ and $R=2675 \mathrm{~mm}$, with an optical dead area due to imperfections of $\sim 0.5 \%$. The $D_{0}$ is out of specification but tolerable. The beryllium substrate is $\sim 3.8 \mathrm{~mm}$ thick, while the glass coating is $\sim 0.4 \mathrm{~mm}$ thick, being thinnest at the center, $\sim 0.3 \mathrm{~mm}$, and up to $\sim 1.0 \mathrm{~mm}$ at the edges. Its size is $\sim 400 \mathrm{~mm} \times \sim 660 \mathrm{~mm}$, weight $\sim 2.7 \mathrm{~kg}$, $\sim 1.6 \% X_{0}$ and $\sim 1 \% \lambda_{\mathrm{I}}$. It is the first ever large sized glass-coated beryllium mirror, and with the refinement of the manufacturing technique, future mirrors of improved optical quality are to be expected.

\section{Carbon-fiber technology}

Carbon fiber reinforced polymer (CFRP) is also used to fabricate light-weight mirrors [3]. CFRP light-weight mirrors were fabricated at $\mathrm{CMA}^{7}$ and consist of $\sim 70 \%$ carbon-fiber $^{8}$ which is the reinforcement material and $\sim 30 \% \operatorname{resin}^{9}$ which is the matrix material that binds the fibers together. The advantages of using CFRP is that it is considerably cheaper than beryllium, there are no safety implications and mirrors are light-weight with an areal density of $\lesssim 6 \mathrm{~g} / \mathrm{m}^{2}$, equivalent to $\lesssim 1.4 \% X_{0}$ and $\lesssim 0.7 \% \lambda_{\mathrm{I}}$. The CFRP mirrors are manufactured by the process called optical replication. Several CFRP sheets and core cells $(50 \mathrm{~mm}$ in diameter) are laid over a finely polished Pyrex glass mandrel matching the CFRP coefficient of thermal expansion. The sheets are laid and glued together in fixed orientations resulting in a quasi-isotropic CFRP bottom skin. Each sheet is unidirectional and a few $100 \mu \mathrm{m}$ thick. On top of the bottom skin, core cells and CFRP sheets forming the top skin, are laid forming a honeycomb sandwich (see Fig. 2). Curing takes place by applying heat and pressure so that the CFRP honeycomb hardens taking the shape of the mandrel and then is separated from it. After appropriately cleaning the front surface the mirror is then ready to be vacuumcoated with a reflective film.

CFRP prototypes manufactured by CMA were successfully tested at CERN [4] for their compatibility with fluorocarbon gas and radiation hardness. The radiation exposure was up to $10 \mathrm{kGy}$ in three separate steps, with a total absorbed dose of 1,4 and $10 \mathrm{kGy}$, respectively, using gammas at a cobalt-60 facility. ${ }^{10}$ The fluorocarbon exposure was approximately one year in duration, with the prototypes placed in a gas tank filled with $\mathrm{C}_{4} \mathrm{~F}_{10}$ at room temperature and with a slight overpressure $(\sim 5 \mathrm{kPa})$ with respect to atmospheric pressure. The CFRP prototypes are: a demonstration mirror to prove the design concept, i.e. square shaped of dimensions $600 \mathrm{~mm} \times 600 \mathrm{~mm}$, and $R=2200 \mathrm{~mm}$; two small spherical mirrors (called M1, M2), $150 \mathrm{~mm}$ in diameter and $R=1890 \mathrm{~mm}$; and two flat CFRP samples (called F1, F2) each $\sim 100 \mathrm{~mm} \times 100 \mathrm{~mm} \times 0.5 \mathrm{~mm}$ without any reflective coating to be used in mechanical tests. All three prototype mirrors have an

\footnotetext{
${ }^{7}$ Composite Mirror Applications, Tucson, AZ, USA.

8 Toray M46J PAN carbon-fibers.

${ }^{9}$ EX-1515 cyanate ester resin, Bryte Technologies.

${ }^{10}$ IONISOS, Dagneux, France.
} 


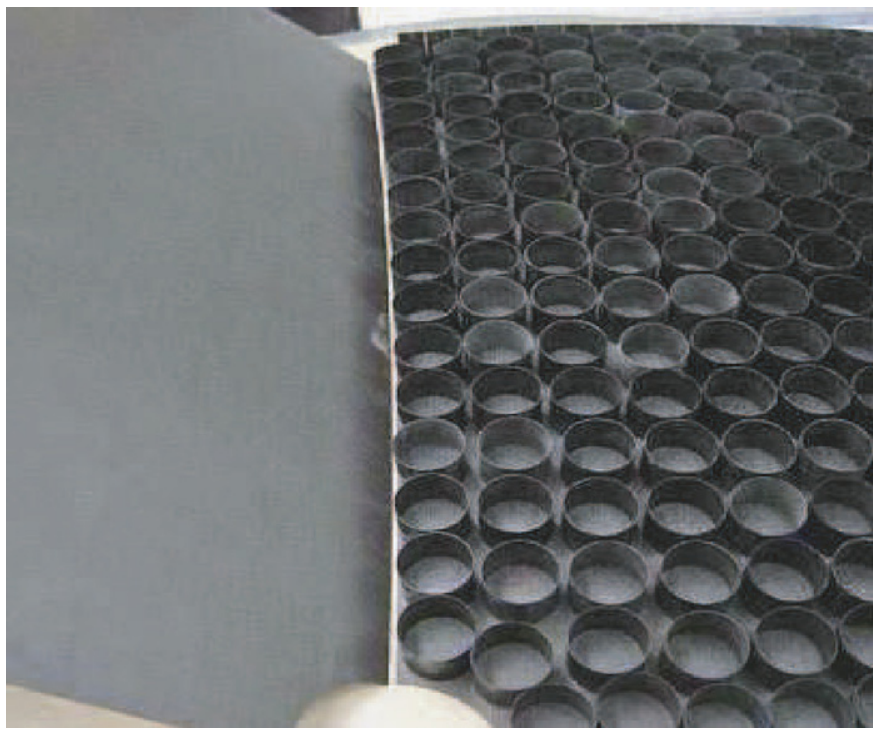

Fig. 2. CFRP mirror sandwich honeycomb structure. The top skin which is glued onto the core cells is seen on the left.

areal density of $\sim 5 \mathrm{~kg} / \mathrm{m}^{2}$ and an aluminium plus silicon monoxide $(\mathrm{Al}+\mathrm{SiO})$ reflective coating. The demonstration mirror, a small mirror (M1) and a flat sample (F1) were exposed only to $\mathrm{C}_{4} \mathrm{~F}_{10}$, while the other small mirror (M2) and flat sample (F2) were exposed only to radiation. No change in the optical and mechanical properties of the prototypes was measured after either exposure. The $D_{0}$ and $\mathrm{R}$ of the demonstration mirror $\left(D_{0} \sim 1 \mathrm{~mm}, R \sim 2205 \mathrm{~mm}\right)$ and the two small mirrors M1 and M2 ( $D_{0} \sim 0.8 \mathrm{~mm}, R \sim 1890 \mathrm{~mm}$ ) remained unchanged, as well as the reflectivity over the $200-600 \mathrm{~nm}$ range. ${ }^{11}$ Also the mechanical properties of the flat samples (F1, F2) remained invariant, i.e. flexural rigidity (bending) $33 \mathrm{~N} / \mathrm{mm}$, tensile rigidity (pulling) $\sim 8 \mathrm{kN} / \mathrm{mm}$ and average surface roughness ${ }^{12}\left(R_{\mathrm{a}}\right) \sim 1.8 \mu \mathrm{m}$. Furthermore, comparison of microscope photos $(\times 100)$ after varying degrees of either exposure showed no noticeable change in the structure of the flat samples.

The CFRP technology proved to be very promising and it was chosen for RICH 1. The optical design consists of four spherical mirrors, $R=2700 \mathrm{~mm}$, supported by a CFRP frame. The frame is made of two C-like halves joined at the center top and bottom, with the $\mathrm{LHCb}$ beampipe passing through the center of the four mirrors, see Fig. 3. Each mirror is $\sim 640 \mathrm{~mm} \times 835 \mathrm{~mm}$ with an areal density of $\sim 5.5 \mathrm{~kg} / \mathrm{m}^{2}\left(\sim 1.3 \% X_{0}\right)$ and is supported by the CFRP frame at three points on the outer mirror corners, which lie outside the detector acceptance. The mirrors have a sandwich honeycomb structure, consisting of two outer CFRP skins with cylindrical core cells lying in between the skins as reinforcement (see Fig. 2). The two outer edges which support the mirror have square cells for increased reinforcement. The core cells and the skins are glued together. FEA ${ }^{13}$ studies have been carried out for the mirrors supported in the RICH 1 configuration, giving a maximum deflection to the unsupported mirror edge (near the beampipe) of $\sim 3.5 \mu \mathrm{m}$.

The four production mirrors were characterized at CERN. The mirrors are of good optical quality having very similar $D_{0}$ and $R$ values between mirrors: i.e. $D_{0} \sim 1.2 \mathrm{~mm}$ and $R \sim 2710 \mathrm{~mm}$. Ronchigrams taken at CMA give straight lines indicating that the mirrors have a good spherical surface. The four production mirrors

\footnotetext{
11 Demonstration mirror too large to measure its reflectivity.

12 The surface is not of optical quality.

${ }^{13}$ Finite element analysis software, SolidWorks.
}

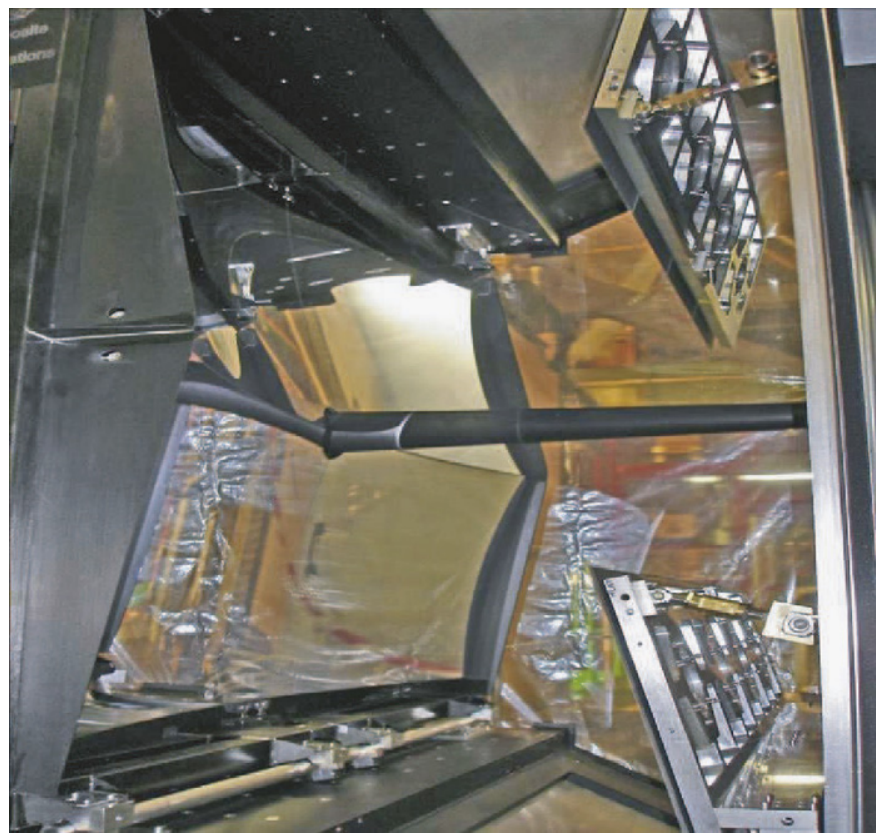

Fig. 3. The four coated CFRP mirrors and CFRP support frame installed in RICH 1 with the beryllium beampipe at the center and two flat glass mirror arrays on the right.

were coated at $\mathrm{SESO}^{14}$ with $\mathrm{Al}(80 \mathrm{~nm})+\mathrm{MgF}_{2}(160 \mathrm{~nm}),{ }^{15}$ giving an average reflectivity of $\sim 90 \%$ with the reflectivity being always $>85 \%$ over the $200-600 \mathrm{~nm}$ range. CMA's standard reflectivity coating is $\mathrm{Al}(80 \mathrm{~nm})+\mathrm{SiO}(80 \mathrm{~nm})$ which gives a good reflectivity in the visible but did not quite meet the LHCb specification in the UV. The CFRP spherical mirrors were installed into RICH 1 in July 2007 in the LHCb underground experiment hall, now awaiting the LHC startup.

\section{Summary}

Two light-weight mirror technologies have been tested successfully: glass-coated beryllium and carbon-fiber (CFRP). The overall optical quality of the full-sized beryllium prototype mirror is good, $D_{0}=3.3 \mathrm{~mm}$ and $R=2675 \mathrm{~mm}$. It is the first ever large-sized beryllium mirror, $\sim 400 \mathrm{~mm} \times \sim 660 \mathrm{~mm}$, with a thin beryllium substrate $\sim 4 \mathrm{~mm}$, and a thin glass coating $\sim 0.4 \mathrm{~mm}$ resulting in $\sim 1.6 \% X_{0}$. Improvement in the quality of future mirrors is to be expected with the refinement of the manufacturing technique. Four large, $\sim 640 \mathrm{~mm} \times 835 \mathrm{~mm}$, CFRP spherical mirrors have been manufactured for the LHCb detector RICH 1 detector. They are of good optical quality, well within requirements: $D_{0} \sim 1.2 \mathrm{~mm}, R \sim 2710 \mathrm{~mm}$, reflectivity $\sim 90 \%(200-600 \mathrm{~nm})$, $\sim 1.3 \% X_{0}$, and prototypes have been tested successfully for radiation hardness and $\mathrm{C}_{4} \mathrm{~F}_{10}$ compatibility. CFRP was chosen for RICH 1 because, as well as meeting all the requirements, was also advantageous on grounds of delivery time and cost.

\section{References}

[1] LHCb Collaboration, CERN/LHCC/2003-030, 2003.

[2] F. Metlica, et al., Nucl. Instr. and Meth. A 570 (2007) 565.

[3] R.C. Romeo, et al., Proc. SPIE 6273 (2006) 62730S.

[4] R.N. Martin, et al., Proc. SPIE 6670 (2007) 667000

\footnotetext{
${ }^{14}$ Societe Europeenne de Systemes Optiques, Aix en Provance, France.

${ }^{15}$ No uniformity coating tool used. The $\mathrm{MgF}_{2}$ thickness varies from $\sim 160 \mathrm{~nm}$ at the center of the mirror to $\sim 80 \mathrm{~nm}$ at its periphery; similarly for the $\mathrm{Al}(80 \mathrm{~nm})$.
} 